\title{
Testosterone replacement in androgen insensitivity: is there an advantage?
}

\author{
Rafael Loch Batista, Berenice Bilharinho Mendonca \\ Faculdade de Medicina FMUSP, Universidade de Sao Paulo, Sao Paulo, SP, Brazil \\ Correspondence to: Berenice Bilharinho Mendonca, MD, PhD. Faculdade de Medicina da Universidade de São Paulo, Av. Dr. Arnaldo, 455 - Cerqueira \\ César, 01246-903 São Paulo, SP, Brazil. Email: beremen@usp.br. \\ Provenance: This is an invited Editorial commissioned by the Section Editor Jianqing Tian (Department of Endocrinology, Xiamen Humanity \\ Hospital, Xiamen, China). \\ Comment on: Birnbaum W, Marshall L, Werner R, et al. Oestrogen versus androgen in hormone-replacement therapy for complete androgen \\ insensitivity syndrome: a multicentre, randomised, double-dummy, double-blind crossover trial. Lancet Diabetes Endocrinol 2018;6:771-80.
}

Submitted Oct 17, 2018. Accepted for publication Oct 30, 2018.

doi: $10.21037 /$ atm.2018.10.73

View this article at: http://dx.doi.org/10.21037/atm.2018.10.73

Androgen insensitivity syndrome (AIS) is the most common etiology of 46,XY disorders of sex development (DSD) (1). In the complete phenotype (CAIS), affected individuals present typically female external genitalia at birth, are assigned as female and present psychosexual development in agreement with sex assignment (2). In CAIS, bilateral gonadectomy is necessary either due to inguinal hernia at childhood or to avoid germ cell tumor development (3). The consequence of bilateral gonadectomy is the need of oestrogen replacement as expected for matched-age women. However, despite an adequate hormonal replacement with estrogens, some CAIS women complained about reduced psychological wellbeing and sexual satisfaction after bilateral gonadectomy $(4,5)$.

Birnbaum et al. designed a clinical trial to answer the question: is testosterone able to improve wellbeing and sexual functioning in patients with CAIS? That question was based on evidence that some sexual behavior brain activation is independent of a functioning androgen receptor and depends on the conversion of testosterone into estrogen by aromatase $(6,7)$.

The effectiveness and safety of testosterone replacement on wellbeing and sexuality in patients with CAIS were evaluated in a national, multicenter, double blind, randomized crossover trial conduced in Germany at three university centers and three specialized treatment institutions (8). The authors enrolled 26 patients with CAIS (aged 18-54 years), with molecular diagnosis of AIS and previous gonadectomy (at least 1 year). They were treated with estradiol $1.5 \mathrm{mg} /$ day during a 2 -month run-in phase. After this phase, they were divided into two groups (14 in sequence $\mathrm{A}$ and 12 in sequence $\mathrm{B}$ ), in a random manner to receive: oestradiol $(1.5 \mathrm{mg} /$ day or testosterone $50 \mathrm{mg} /$ day for 6 months (sequence A). Gel preparations for both hormonal replacements via transdermal application were used daily. After that period, there was a cross over and the groups changed from the previous hormone-replacement therapy to the other one, in a double-blinded manner for more 6 months (sequence B).

The primary endpoint was mental health and secondary endpoints were psychological wellbeing and sexual functioning. Safety parameters were also analyzed, and possible side effects were reported and classified according to severity into three categories: mild, moderate, and intense.

The health-related quality of life (HRQoL) and mental HRQoL (MHRQoL) were measured with the standardized SF-36 German version, a multipurpose and validated shortform survey which measure eight health domains (physical functioning, vitality, social functioning, role-physical, roleemotional, bodily pain, mental health, general health) and psychometrical physical and mental health summary measures. The Brief Symptom Inventory (BSI), which is an instrument that evaluates psychological distress and psychiatric disorders covering nine symptom dimensions, was used to assess psychological wellbeing. Sexual functioning was measured with a German version of the Female Sexual Function Index (FSFI), a multidimensional 
self-report instrument for assessing the key dimensions of sexual function in women. This questionnaire provides scores on six domains of sexual function (desire, arousal, lubrication, orgasm, satisfaction, and pain) as well as a total score.

Testosterone, estradiol, LH, FSH and urinary steroid metabolites were measured at baseline, after the runin phase and during the treatment, both for treatment monitoring and for compliance estimative.

There was no significant difference in the effect of oestradiol and testosterone on mental health scores or physical summary scores. The MHRQoL and physical HRQoL scores did not differ significantly from baseline either for testosterone or oestradiol replacement. Wellbeing evaluated by BSI scores showed that the patients enrolled had psychological distress at beginning of the trial. Nevertheless, there's no significant difference regarding BSI scores for psychological wellbeing between two groups.

With regard to sexual functioning, the mean value of FSFI score at baseline was 15.78 , which is consistent with a low prevalence of satisfactory sexual functioning among those CAIS individuals before treatment. Both hormonereplacement therapies increase the FSFI score from baseline to 19.3 (under oestradiol) and to 21.5 (under testosterone). These increments in FSFI scores were significant from basal FSFI score but it was not significant between each other. The mean FSFI total scores for sexual functioning were higher for testosterone than oestradiol treatment in all sexual domains (except by sexual satisfaction) but statistical significance only occurred in the desire domain $(\mathrm{P}=0.018)$.

Regarding safety, three patients dropped out because of adverse events. One patient had fibrous mastopathy receiving oestradiol during run in phase (classified as a serious adverse event). The other two side effects (episodes of depression and hot flush symptoms) occurred in patients receiving testosterone.

In summary, at the end of the study the difference of FSFI total score between both groups was not significant. Although, testosterone was superior to oestradiol in the improvement of sexual desire in the FSFI sub analysis (analysis of each domain) there were no differences on safety between both treatments.

The authors concluded that testosterone was well tolerated and safety as estradiol and it could be an alternative hormone-replacement therapy for patients with CAIS, especially when sexual desire is reduced.

It has been demonstrated that sexual life of $46, \mathrm{XY}$ DSD people is affected for many reasons. However, the results of studies on quality of sexual life are inconsistent, in part either due to the lack of questionnaire on sexual life specific for DSD and for the heterogeneity of the DSD etiologies enrolled in previous studies $(5,9,10)$.

The Birnbaum et al. clinical trial is very interesting because it is the first clinical trial designed for disorders of sexual development individuals. In addition to contributing results of efficacy, this study expands knowledge about the role of androgens in human sexuality. However, it is necessary to pay attention to some points: the sexual improvement by testosterone seems to be related to sexual desire, which was not enough to improve sexual satisfaction. The benefits of steroid replacement in bone integrity and cardiovascular protection are well-established with estrogen replacement but it is unknown regarding testosterone. The shorter period of therapy (6 months) preclude the conclusion of long-term safety.

Although testosterone could be an alternative hormone therapy for patients with CAIS, long term follow-up, assessment of impact on other psychological issues and on bone metabolism and cardiovascular protection are necessary to consider testosterone replacement in CAIS in clinical practice.

\section{Acknowledgements}

None.

\section{Footnote}

Conflicts of Interest: The authors have no conflicts of interest to declare.

\section{References}

1. Hughes IA, Davies JD, Bunch TI, et al. Androgen insensitivity syndrome. Lancet 2012;380:1419-28.

2. Batista RL, Costa EMF, Rodrigues AS, et al. Androgen insensitivity syndrome: a review. Arch Endocrinol Metab 2018;62:227-35.

3. Döhnert U, Wünsch L, Hiort O. Gonadectomy in Complete Androgen Insensitivity Syndrome: Why and When? Sex Dev 2017;11:171-4.

4. Köhler B, Kleinemeier E, Lux A, et al. Satisfaction with genital surgery and sexual life of adults with XY disorders of sex development: results from the German clinical evaluation study. J Clin Endocrinol Metab 2012;97:577-88.

5. Minto CL, Liao KL, Conway GS, et al. Sexual function in 
women with complete androgen insensitivity syndrome. Fertil Steril 2003;80:157-64.

6. Wu MV, Manoli DS, Fraser EJ, et al. Estrogen masculinizes neural pathways and sex-specific behaviors. Cell 2009;139:61-72.

7. Nugent BM, Wright CL, Shetty AC, et al. Brain feminization requires active repression of masculinization via DNA methylation. Nat Neurosci 2015;18:690-7.

8. Birnbaum W, Marshall L, Werner R, et al. Oestrogen versus androgen in hormone-replacement therapy for

Cite this article as: Batista RL, Mendonca BB. Testosterone replacement in androgen insensitivity: is there an advantage? Ann Transl Med 2018;6(Suppl 1):S85. doi: 10.21037/atm.2018.10.73 complete androgen insensitivity syndrome: a multicentre, randomised, double-dummy, double-blind crossover trial. Lancet Diabetes Endocrinol 2018;6:771-80.

9. Schönbucher V, Schweizer K, Rustige L, et al. Sexual quality of life of individuals with 46,XY disorders of sex development. J Sex Med 2012;9:3154-70.

10. Schonbucher V, Schweizer K, Richter-Appelt H. Sexual quality of life of individuals with disorders of sex development and a 46,XY karyotype: a review of international research. J Sex Marital Ther 2010;36:193-215. 